\title{
Wichtige epidemiologische Studientypen
}

\author{
- Artikel Nr. 18 der Statistik-Serie in der DMW -
}

\author{
Common study designs in epidemiology
}

\author{
Autoren \\ S. J. Klug ${ }^{1}$ R. Bender ${ }^{2}$ M. Blettner ${ }^{1}$ S. Lange ${ }^{2}$ \\ Institut \\ ${ }^{1}$ Institut für Medizinische Biometrie, Epidemiologie und Informatik (IMBEI), \\ Klinikum der Johannes Gutenberg-Universität Mainz \\ 2 Institut für Qualität und Wirtschaftlichkeit im Gesundheitswesen, Köln
}

Die Epidemiologie ist die Lehre von der Verteilung der Krankheiten in der Bevölkerung. Diese Krankheitsverteilung (deskriptive Epidemiologie) und die Assoziation zwischen einzelnen Krankheiten und verschiedenen Risikofaktoren (analytische Epidemiologie) können in epidemiologischen Studien untersucht werden. Die wichtigsten Studientypen der analytischen Epidemiologie sind Kohortenstudien, oder auch Längsschnittstudien genannt, Fall-Kontroll-Studien und Querschnittstudien (Surveys) $[10,11,15,19,24]$. Diese unterschiedlichen, beobachtenden Studiendesigns kommen in Abhängigkeit von Fragestellung und Studienziel zur Anwendung (Tab. 1, Abb. 1). Bei allen drei Studientypen können zusätzlich zur Datenerhebung mit epidemiologischen Fragebogen und Interviews (klassische Epidemiologie) auch biologische Materialien, wie z. B. Blutproben der Studienteilnehmer und Studienteilnehmerinnen gesammelt werden. Biologische Materialien oder Biomarker werden zunächst mit molekularbiologischen Methoden im Labor analysiert und anschließend in Verbindung mit den epidemiologischen Daten statistisch ausgewertet (Molekulare Epidemiologie) $[21,23]$.

\section{Kohortenstudien}

\section{$\checkmark$}

Kohortenstudien sind Längsschnittstudien über die Zeit. Individuen werden bezüglich einer Exposition, wie z. B. Rauchen oder einer beruflichen Tätigkeit,

\begin{tabular}{|c|}
\hline Vergangenheit Gegenwart \\
Retrospektive Kohortenstudie \\
Quentroll-Studie \\
\hline
\end{tabular}

Abb. 1 Darstellung des zeitlichen Ablaufs der Datenerhebung bei den verschiedenen Studientypen. ${ }^{*}$ In retrospektiven Kohortenstudien, bei denen die Exposition retrospektiv erfasst wurde, kann das Follow-up zur Erfassung des möglichen Eintretens der zu untersuchenden Krankheiten oder Todesursachen auch prospektiv durchgeführt werden. ausgewählt und über die Zeit beobachtet, um Exponierte mit Nicht-Exponierten bezüglich der Inzidenz von Krankheiten (Inzidenzstudie) oder der auftretenden Todesursachen (Mortalitätsstudie) zu vergleichen. Bei einer prospektiven Kohortenstudie werden Exposition und andere Kovariablen zu Beginn der Studie, also vor dem möglichen Eintritt der Erkrankung erhoben. Personen, die bei der BaselineUntersuchung an einer der Zielerkrankungen erkrankt sind, werden aus der Studienkohorte ausgeschlossen. Die Kohorte wird anschließend meist über Jahre beobachtet und auftretende Erkrankungen und Todesfälle dokumentiert (Follow-up).

Einen Sonderfall stellt die retrospektive oder historische Kohortenstudie dar, die vor allem in der Arbeitsepidemiologie Anwendung findet. Hier werden Expositionen aus der Vergangenheit erhoben und anschließend die Kohorte für Erkrankungen oder Todesursachen verfolgt.

Ein weiterer Sonderfall der Kohortenstudien sind Interventionsstudien, die der experimentellen Epidemiologie zuzuordnen sind. Beispiele hierfür sind gemeindebezogene Interventionsstudien und auch randomisierte klinische Studien.

\section{Analyse \\ $\checkmark$}

Durch den Vergleich der Anzahl der beobachteten Erkrankungsfälle unter den exponierten Personen in der Kohorte mit einer nicht-exponierten Bezugsbevölkerung (externe Analyse) können Standardisierte Mortalitäts-Ratios (SMR) oder Standardisierte Inzidenz-Ratios (SIR) berechnet werden [9]. SMR und SIR sind zumeist für Alter und Geschlecht standardisiert. Über den Vergleich zwischen exponierten Personen mit nicht-exponierten Personen innerhalb der Kohorte (interne Analyse) können rohe relative Risiken (RR) über Häufigkeitstabellen geschätzt werden [2]. Ein wichtiger Aspekt bei der Auswertung von Kohortenstudien ist allerdings in der Regel die Berücksichtigung von Einflüssen ande-
Schlüsselwörter

Epidemiologie

Studiendesign

Kohortenstudie

Fall-Kontroll-Studie

Key words

Epidemiology

Study design

$\checkmark$ Cohort study

$\checkmark$ Case-control study

Bibliografie

Dol $10.1055 / \mathrm{s}-2007-959041$ Dtsch Med Wochenschr 2007; 132: e45-e47 - (c) Georg Thieme Verlag KG Stuttgart - New York . ISSN 0012-0472

Korrespondenz

Dr. rer. nat. Stefanie Klug

Institut für Medizinische Biometrie, Epidemiologie und Informatik (IMBEI), Klinikum der Johannes Gutenberg-Universität Mainz

55101 Mainz

eMail klug@imbei.uni-mainz.de 
Tab. 1 Übersicht über Charakteristika der wichtigsten epidemiologischen Studientypen.

$\begin{array}{llll}\text { Studientyp } & \begin{array}{l}\text { Studienpopulation/ } \\ \text { Auswahlkriterien }\end{array} & \begin{array}{l}\text { Daten- } \\ \text { erhebung }\end{array} & \text { Effektmaße } \\ \text { Kohortenstudie } & \begin{array}{l}\text { Stichproben aus Expo- } \\ \text { nierten und Nicht- } \\ \text { Exponierten }\end{array} & \begin{array}{l}\text { Prospektiv oder } \\ \text { retrospektiv }\end{array} & \begin{array}{l}\text { Inzidenz, } \\ \text { RR, SMR, SIR }\end{array} \\ \begin{array}{l}\text { Fall-Kontroll- } \\ \text { Studie }\end{array} & \begin{array}{l}\text { Inzidente Fälle (Kranke) } \\ \text { und definierte Kontrollen } \\ \text { (Gesunde) }\end{array} & \text { retrospektiv } & \text { OR } \\ \begin{array}{llll}\text { Querschnitt- } \\ \text { studie }\end{array} & \begin{array}{l}\text { Repräsentative Stich- } \\ \text { probe aus der Zielpopu- }\end{array} & \begin{array}{l}\text { Stichtermin, } \\ \text { retrospektiv }\end{array} & \text { Prävalenz } \\ & \text { lation } & & \\ & & & \end{array}$

rer Variablen, z. B. Störgrößen (confounder), um eine mögliche Verzerrung (bias) bei der Effektschätzung so weit wie möglich zu reduzieren [3]. Werden alle Kohortenmitglieder gleich lange beobachtet, kommt als Auswertungsmethode die logistische Regression in Frage [4]. Bei unterschiedlich langen Follow-up Zeiten der Kohortenmitglieder entstehen zensierte Daten, die mit Methoden für Überlebenszeiten analysiert werden [26]. Adjustierte relative Risiken können mit Hilfe der Poisson Regression und adjustierte Hazard Ratios (HR) mit Hilfe des Cox Proportional Hazards Modells berechnet werden $[9,19,27]$.

\section{Vor- und Nachteile \\ $\checkmark$}

Große epidemiologische Kohortenstudien liefern meist Daten hoher Qualität und Ergebnisse mit hoher Repräsentativität. Die zeitliche Sequenz von Exposition und Erkrankung ist eindeutig und dieser Studientyp ist auch für seltene Expositionen geeignet. Aussagen zu möglichen kausalen Zusammenhängen von Exposition und Erkrankung können nur aus solchen großen prospektiven Kohortenstudien abgeleitet werden, und auch hier nur unter Berücksichtigung der Kriterien von Bradford Hill [12]. Die Nachteile von Kohortenstudien sind hohe Kosten und lange Dauer, auch sind sie für die Untersuchung seltener Erkrankungen ungeeignet.

\section{Beispiele}

Ein Beispiel für eine prospektive Kohortenstudie ist die internationale EPIC Studie (European Prospective Investigation into Cancer). Hier wurden in neun europäischen Ländern, darunter auch an zwei Standorten in Deutschland (Potsdam und Heidelberg), ca. 400000 Personen nach festgelegten Ein- und Ausschlusskriterien in die Studie aufgenommen. Untersucht wird der Zusammenhang zwischen Expositionen, wie Ernährungsgewohnheiten und Lebensstilfaktoren, und künftigen Krebserkrankungen [7,17]. Die Personen in der Studienkohorte mussten zur Erhebung der Exposition bei der Baseline-Untersuchung (erster Studienkontakt) und bei den verschiedenen Follow-up Untersuchungen epidemiologische Fragebogen (study questionnaire) und spezielle Ernährungsfragebögen (food frequency questionnaire) ausfüllen. Ausserdem wurden Blutproben entnommen, die zentral gelagert und analysiert werden. Die Inzidenz und Mortalität der Kohorte für unterschiedliche Krebserkrankungen wird in der Zukunft beobachtet.

In der Arbeitsepidemiologie werden hauptsächlich retrospektive Kohortenstudien durchgeführt. Die Exposition der Studienkohorte fand in der Vergangenheit statt. Ein Beispiel ist eine kürzlich abge- schlossene internationale Studie beim fliegenden Personal bei verschiedenen europäischen Luftfahrtgesellschaften. Hier wurde der Zusammenhang zwischen kosmischer Strahlung und Krebserkrankungen untersucht [5,6,25]. Die Daten zur Exposition von mehr als 26000 Piloten und Kabinenpersonal wurden aus den archivierten Datenbeständen der Luftfahrtgesellschaften abstrahiert und die Mortalität wurde über ein Follow-up erhoben. Berufliche Expositionen scheinen keine starken Auswirkungen auf die Mortalität in dieser Kohorte zu haben.

Bei beiden Beispielen handelt es sich um multizentrische Studien, die durch eine große Studienkohorte zu präzisen Schätzungen führen und in ihren Ergebnissen internationale Gültigkeit aufweisen.

\section{Fall-Kontroll-Studien} $\nabla$

In Fall-Kontroll-Studien werden neuerkrankte Personen (inzidente Fälle) mit nicht erkrankten Personen (Kontrollen) bezüglich verschiedener Risikofaktoren (Exposition) verglichen. Die Fälle werden zumeist aus Krankenhäusern rekrutiert. Die Kontrollen können entweder aus demselben Krankenhaus wie die Fälle stammen (Krankenhauskontrollen) oder eine Zufallstichprobe der Bevölkerung aus dem Einzugsgebiet des Krankenhauses darstellen (Bevölkerungskontrollen). In jedem Fall hat die Auswahl der Kontrollen mit großer Sorgfalt zu erfolgen, um Selektionsfehler zu vermeiden. Fälle und Kontrollen werden meist für Geschlecht und Alter angepasst (matching).

\section{Analyse \\ $\nabla$}

Fall-Kontroll-Studien werden deskriptiv mit Hilfe von Vierfeldertafeln ausgewertet [2]. Durch Stratifizierung und der Anwendung der Mantel-Haenszel-Methode können einige wenige kategorielle Variablen, z. B. Confounder wie Alter, berücksichtigt werden [8]. Bei Vorliegen mehrerer Confounder, die auch stetiges Messniveau besitzen können, kommt die Anwendung der logistischen Regression in Frage $[4,8]$. Das berechnete Effektmaß ist das Odds Ratio (OR) [2]. Bei seltenen Erkrankungen gilt, dass das OR in etwa dem relativen Risiko (RR) entspricht. Fall-Kontroll-Studien, bei denen Fälle und Kontrollen z. B. für Alter im Studiendesign aneinander angepasst wurden (matching), werden mit Hilfe der bedingten logistischen Regression ausgewertet [8].

\section{Vor- und Nachteile \\ $\nabla$}

Fall-Kontroll-Studien sind besonders für seltene Erkrankungen geeignet und sie sind weniger zeit- und kostenintensiv als Kohortenstudien. Die Auswahl der Kontrollgruppe ist oft problematisch und die zeitliche Abfolge von Exposition und Erkrankung unklar. Durch Fall-Kontroll-Studien können keine Aussagen zur Inzidenz einer Erkrankung getroffen werden.

\section{Beispiele}

Durch eine Serie von Fall-Kontroll-Studien, die weltweit durchgeführt wurden, erhärtete sich der Verdacht, das eine Infektion mit humanen Papillomaviren (HPV) den Hauptrisikofaktor für die Entstehung eines Zervixkarzinom darstellt $[16,18,20]$. Inzidente Fälle und Krankenhauskontrollen wurden in einem fragebogengestützten Interview zu Risikofaktoren, wie reproduktivem und sexuellem Verhalten, zu Früherkennungsuntersuchungen, sowie zu sozio-demographischen Informationen befragt. Tumorbiopsien und zervikale Zellen wurden mit molekularbiologischen Methoden auf eine HPV 
Infektion untersucht. Das Risiko einer mit einem bestimmten HPV Typ, wie z. B. HPV 16 oder 18 infizierten Frau an einem Zervixkarzinom zu erkranken, war stark erhöht. Die Ergebnisse dieser Fall-Kontroll-Studien wurden von molekularbiologischen Experimenten gestützt und in Kohortenstudien bestätigt, wodurch heute die Aussage zulässig ist, dass HPV eine notwendige Ursache für die Entstehung eines Zervixkarzinoms ist [14]

\section{Querschnittstudien}

$\checkmark$

Querschnittstudien umfassen eine definierte Auswahl von Personen aus der festgelegten Zielpopulation und ermitteln Expositionen und Erkrankungen gleichzeitig zu einem festen Stichtermin. Da aber häufig lange Zeit zwischen Exposition und Erkrankung liegt, ist dieser Studientyp in der analytischen Epidemiologie zum Kausalitätsnachweis nur sehr bedingt einsetzbar. Querschnittstudien sind daher hauptsächlich ein Instrument der deskriptiven Epidemiologie, wo sie besonders der Hypothesengenerierung dienen.

\section{Analyse}

$\checkmark$

Die Prävalenz von Erkrankungen, Risikofaktoren oder von bestimmten Gesundheitsverhalten kann durch relative Häufigkeiten geschätzt werden.

\section{Vor- und Nachteile}

$\checkmark$

Querschnittstudien sind relativ schnell und bei vergleichsweise niedrigen Kosten durchführbar. Sie liefern oft gute Hinweise auf Risikofaktoren, allerdings bleibt die zeitliche Sequenz zwischen Exposition und Erkrankung unklar. Querschnittstudien sind zudem für seltene Erkrankungen und seltene Expositionen ungeeignet.

\section{Beispiel}

\section{$\checkmark$}

Von 1997 bis 1999 wurde das erste gesamtdeutsche Gesundheitssurvey (Bundesgesundheitssurvey) durchgeführt $[1,22]$. Insgesamt nahmen 7124 Personen daran teil, die einen epidemiologischen Fragebogen ausfüllten, ärztlich untersucht wurden und eine Blut- und Urinprobe abgaben. Ein Ergebnis dieser umfangreichen Querschnittstudie war zum Beispiel, dass lediglich 22,6\% der Männer und 36,5\% der Frauen im Jahre 1997 an Krebsfrüherkennungsuntersuchungen teilgenommen haben [13].

\section{kurzgefasst}

Die wichtigsten epidemiologischen Studientypen sind Kohortenstudien, Fall-Kontroll-Studien und Querschnittstudien. Je nach Fragestellung und Datenlage erlauben diese verschiedenen Studientypen die Untersuchung des Zusammenhangs von Expositionen und Erkrankungen (Kohortenstudien und Fall-Kontroll-Studien) oder beschreiben Prävalenzen (Querschnittstudie).

Dieser Beitrag ist eine überarbeitete Fassung aus dem Supplement Statistik aus dem Jahr 2004.
Literatur

1 Bellach BM, Knopf H, Thefeld W. Der Bundesgesundheitssurvey 1997/98. Gesundheitswesen 1999; 60: 59-68

2 Bender R, Lange S. Die Vierfeldertafel. Dtsch Med Wochenschr 2007; 132: e12-e14

3 Bender R, Ziegler A, Lange S. Multiple Regression. Dtsch Med Wochenschr 2007; 132: e30-e32

4 Bender R, Ziegler A, Lange S. Logistische Regression. Dtsch Med Wochenschr 2007; 132: e33-e35

5 Blettner M, Zeeb H, Langner I, Schafft T. Mortality from cancer and other causes among airline cabin attendants in Germany, 1960 - 1997. Am J Epidemiol 2002; 156: 556-565

6 Blettner M, Zeeb H, Langner I, Hammer GP. Epidemiologische Studie beim fliegenden Personal der Deutschen Lufthansa und der LTU. Dt Ärzteblatt 2002; 51/52: A3462-A3469

7 Boeing H, Korfmann A, Bergmann MM. Recruitment procedures of EPICGermany. European Investigation into Cancer and Nutrition. Ann Nutr Metab 1999; 43: 205-215

8 Breslow NE, Day NE. Statistical methods in cancer research. The analysis of case-control studies. Lyon: International Agency for Research on Cancer. 1980; Vol. I

9 Breslow NE, Day NE. Statistical methods in cancer research. The analysis of cohort studies. Lyon: International Agency for Research on Cancer, 1987; Vol. II

10 Gail MH, Benichou J. Encyclopedia of Epidemiologic Methods. Chichester, New York, Weinheim, Brisbane, Singapore, Toronto: John Wiley \& Sons, Ltd, 2000

11 Gordis L. Epidemiologie. Marburg: Kilian, 2001

12 Hill $A B$. The Environment and Disease: Association or Causation? Proc $\mathrm{R}$ Soc Med 1965; 58: 295-300

13 Kahl H, Hölling H, Kamtsiuris P. Inanspruchnahme von Früherkennungsuntersuchungen und Maßnahmen zur Gesundheitsförderung. Gesundheitswesen 1999; 61: 163-168

14 Klug SJ, Blettner M. Zervixkarzinom, HPV-Infektion und Screening Stand der Dinge und Zukunftsperspektiven. Dtsch Ärzteblatt 2003; 3: A132-A136

15 Kreienbrock L, Schach S. Epidemiologische Methoden. Stuttgart: Gustav Fischer Verlag, 1997

16 Munoz N, Bosch FX, de Sanjose S, Tafur L, Izarzugaza I, Gili M, Viladiu P, Navarro C, Martos C, Ascunce N. The causal link between human papillomavirus and invasive cervical cancer: a population-based case-control study in Colombia and Spain. Int J Cancer 1992; 52: 743-749

17 Riboli E, Kaaks R. The EPIC Project: rationale and study design. European Prospective Investigation into Cancer and Nutrition. Int J Epidemiol 1997; 26 (Suppl 1): S6-14

18 Rolon PA, Smith JS, Munoz N, Klug SJ, Herrero R, Bosch X, Llamosas F, Meijer CJ, Walboomers JM. Human papillomavirus infection and invasive cervical cancer in Paraguay. Int J Cancer 2000; 85: 486-491

19 Rothman KJ, Greenland S. Modern Epidemiology. Philadelphia, Pa.: Lippincott Williams \& Wilkins, 1998

20 Santos C, Munoz N, Klug SJ, Almonte M, Guerrero I, Alvarez M, Velarde C, Galdos O, Castillo M, Walboomers J, Meijer C, Caceres E. HPV types and cofactors causing cervical cancer in Peru. Br J Cancer 2001; 85: 966-971

21 Schulte P, Perera FP. Molecular Epidemiology. San Diego: Academic Press, 1993

22 Thefeld W, Stolzenberg H, Bellach BM. Bundes-Gesundheitssurvey: Response, Zusammensetzung der Teilnehmer und Non-Responder-Analyse. Gesundheitswesen 1999; 61: S57-S61

23 Toniolo P, Boffetta P, Shuker DEG, Rothmann NHB, Pearce N. Application of Biomarkers in Cancer. Lyon: International Agency for Research on Cancer, 1997

24 Wahrendorf J. Grundbegriffe der Epidemiologie. Stuttgart: Gustav Fischer Verlag, In: Lehrbuch der Hygiene. Sonntag HG, Gundermann KO, Rüden H. (Editoren). 1991

25 Zeeb H, Blettner M, Hammer G, Langner I. Cohort Mortality Study of German Cockpit Crew, 1960 - 97. Epidemiology 2002; 13: 693-699

26 Ziegler A, Lange S, Bender R. Überlebenszeitanalyse: Eigenschaften und Kaplan-Meier-Methode. Dtsch Med Wochenschr 2007; 132: e36-e38

27 Ziegler A, Lange S, Bender R. Überlebenszeitanalyse: Die Cox-Regression. Dtsch Med Wochenschr 2007; 132: e42-e44 